\title{
Damage to the Salience Network and Interactions with the Default Mode Network
}

\author{
Sagar R. Jilka, ${ }^{1,2}$ Gregory Scott, ${ }^{1}$ Timothy Ham, ${ }^{4}$ Alan Pickering, ${ }^{2}$ Valerie Bonnelle, ${ }^{5}$ Rodrigo M. Braga, ${ }^{1,3}$ Robert Leech, ${ }^{1}$ \\ and David J. Sharp ${ }^{1}$ \\ ${ }^{1}$ Computational, Cognitive and Clinical Neuroimaging Laboratory, Centre for Neuroscience, Division of Experimental Medicine, Imperial College London, \\ London, W12 0NN, United Kingdom, ${ }^{2}$ Department of Psychology, Goldsmiths College, University of London, SE14 6NW, United Kingdom, ${ }^{3}$ MRC Clinical \\ Sciences Centre, Faculty of Medicine, Imperial College London, Hammersmith Hospital Campus, London, W12 0NN, United Kingdom, ${ }^{4}$ Systems and \\ Restorative Neurology, University of Cambridge Neurology Unit, Herchel Smith Building for Brain and Mind Sciences Robinson Way, Cambridge, CB2 0SZ, \\ United Kingdom, and ${ }^{5}$ Oxford University, Department of Experimental Psychology, Oxford, OX1 3UD, United Kingdom
}

Interactions between the Salience Network (SN) and the Default Mode Network (DMN) are thought to be important for cognitive control. However, evidence for a causal relationship between the networks is limited. Previously, we have reported that traumatic damage to white matter tracts within the SN predicts abnormal DMN function. Here we investigate the effect of this damage on network interactions that accompany changing motor control. We initially used fMRI of the Stop Signal Task to study response inhibition in humans. In healthy subjects, functional connectivity (FC) between the right anterior insula (rAI), a key node of the SN, and the DMN transiently increased during stopping. This change in FC was not seen in a group of traumatic brain injury (TBI) patients with impaired cognitive control. Furthermore, the amount of SN tract damage negatively correlated with FC between the networks. We confirmed these findings in a second group of TBI patients. Here, switching rather than inhibiting a motor response: (1) was accompanied by a similar increase in network FC in healthy controls; (2) was not seen in TBI patients; and (3) tract damage after TBI again correlated with FC breakdown. This shows that coupling between the rAI and DMN increases with cognitive control and that damage within the SN impairs this dynamic network interaction. This work provides compelling evidence for a model of cognitive control where the $\mathrm{SN}$ is involved in the attentional capture of salient external stimuli and signals the DMN to reduce its activity when attention is externally focused.

Key words: Default Mode Network; functional connectivity; psychophysiological interactions; salience network; traumatic brain injury

\section{Introduction}

Efficient behavior requires the coordinated activity of large-scale brain networks. Interactions between the Salience Network (SN) and the Default Mode Network (DMN) are thought to be important for cognitive control (Fransson, 2005; Kelly et al., 2008; Sridharan et al., 2008; Menon and Uddin, 2010; Bonnelle et al., 2012). The SN responds to external events that are behaviorally salient (Seeley et al., 2007), whereas the DMN shows high activity when subjects have an internal focus of attention, such as during internally directed thought (Gusnard et al., 2001; Buckner et al.,

Received Feb. 5, 2014; revised May 6, 2014; accepted May 28, 2014.

Author contributions: S.R.J., T.H., and D.J.S. designed research; S.R.J., T.H., V.B., and D.J.S. performed research; S.R.J., R.M.B., and R.L. contributed unpublished reagents/analytic tools; S.R.J., G.S., A.P., V.B., R.L., and D.J.S. analyzed data; S.R.J. and D.J.S. wrote the paper.

This work was supported by the Medical Research Council (United Kingdom) and the National Institute of Health Research Professorship (RP-011-048).

The authors declare no competing financial interests.

This article is freely available online through the $J$ Neurosci Author Open Choice option.

Correspondence should be addressed to Prof. David J. Sharp, Computation, Cognitive and Clinical Neuroimaging Laboratory, 3rd Floor, Burlington Danes Building, Hammersmith Hospital, Du Cane Road, London, W12 0NN, United Kingdom. E-mail: david.sharp@imperial.ac.uk.

DOI:10.1523/JNEUROSCI.0518-14.2014

Copyright $\odot 2014$ Jilka et al.

This is an Open Access article distributed under the terms of the Creative Commons Attribution License (http://creativecommons.org/licenses/by/3.0), which permits unrestricted use, distribution and reproduction in any medium provided that the original work is properly attributed.
2008). Changing from automatic behavior, where attention is often focused internally, to behavior guided by external events is accompanied by increased activation within the $\mathrm{SN}$ and deactivation of the DMN (Sharp et al., 2011). One model of cognitive control proposes that the right anterior insula (rAI), a key node of the SN, causally influences activity in other networks, including the DMN (Sridharan et al., 2008; Chiong et al., 2013). However, it is unclear whether the anticorrelations frequently observed between the $\mathrm{SN}$ and DMN are indicative of a causal interaction between the networks.

We have previously studied the change from relatively automatic to controlled behavior using the Stop Signal Task (SST) (Sharp et al., 2010; Bonnelle et al., 2012). Here, subjects are presented with an unexpected "Stop" signal and must attempt to inhibit their responses. Efficient stopping is associated with activation within a right lateralized part of the $\mathrm{SN}$ and deactivation within the DMN (see Fig. 2) (Sharp et al., 2010; Chen et al., 2013). The effects of damage to these networks can be studied after traumatic brain injury (TBI). Previously, we have shown that problems in stopping efficiently after TBI are accompanied by a failure to deactivate the DMN (see Fig. 2) and that damage to the white matter tract within the SN that connects the rAI to the midline presupplementary motor area/dorsal anterior cingulate cortex (rAI-preSMA/dACC tract) is a strong predictor of failure to appropriately deactivate the DMN (Bonnelle et al., 2012). 
Table 1. Neuropsychological results for patients and controls ${ }^{a}$

\begin{tabular}{lccc}
\hline Cognitive variable & Control group & TBI Group 1 & TBI Group 2 \\
\hline Similarities & $35.1 \pm 6.2$ & $38.7 \pm 3.8^{*}$ & $34.8 \pm 5.2$ \\
Matrix Reasoning & $26.5 \pm 4.2$ & $27.4 \pm 4.8$ & $24.9 \pm 6.2$ \\
Verbal Fluency Letter Fluency & $48.5 \pm 12.0$ & $44.1 \pm 11.2$ & $35.9 \pm 14.3^{*}$ \\
Stroop Color Naming (s) & $32.2 \pm 14.1$ & $34.0 \pm 8.9$ & $35.6 \pm 8.5$ \\
Stroop Word Reading (s) & $29.6 \pm 5.1$ & $23.4 \pm 4.8$ & $24.6 \pm 6.5$ \\
Stroop Inhibition (s) & $22.4 \pm 4.2$ & $58.3 \pm 20.9^{* *}$ & $56.9 \pm 18.1^{* *}$ \\
Stroop Inhibition-Switching (s) & $51.5 \pm 18.7$ & $68.4 \pm 21.2^{* *}$ & $66.8 \pm 19.5^{* *}$ \\
Trail Making Test A (s) & $21.5 \pm 5.7$ & $27.4 \pm 10.4^{*}$ & $30.3 \pm 14.1^{* *}$ \\
Trail Making Test B (s) & $53.7 \pm 38.5$ & $64.7 \pm 35.1$ & $67.4 \pm 44$ \\
Trail Making Test Switch Cost & $29.3 \pm 31.3$ & $33.2 \pm 6.6$ & $35.9 \pm 8.2$ \\
Digit Span forward & $11.2 \pm 2.0$ & $10.6 \pm 2.2$ & $9.6 \pm 2.2^{* *}$ \\
Digit Span backward & $7.6 \pm 1.7$ & $7.4 \pm 2.3$ & $7.3 \pm 2.5$ \\
Logical Memory I 1st recall total & $28.1 \pm 8.4$ & $27.9 \pm 6.5$ & $25.7 \pm 7.9$ \\
Logical Memory I recall total & $45.1 \pm 12.8$ & $45.5 \pm 8.7$ & $41.6 \pm 11.5$ \\
Logical Memory II recall total & $26.6 \pm 8.5$ & $29.4 \pm 7.0$ & $25.2 \pm 9.6$ \\
People Test immediate total & $27.5 \pm 6.2$ & $24.1 \pm 5.6$ & $21.6 \pm 7.5^{* *}$ \\
People Test delayed total & $9.2 \pm 3.6$ & $8.8 \pm 2.3$ & $7.6 \pm 4$
\end{tabular}

${ }^{a}$ Data are mean \pm SD. Neuropsychological results for TBI patients compared with an age-matched control group. Stroop test refers to the D-KEFS Color-Word Interference Test.

${ }^{*} p<0.05,{ }^{* *} p<0.005$, significant differences between patients and controls.

Here, we extend this work by investigating whether damage to the rAI-preSMA/dACC tract impairs dynamic interactions between the DMN and SN after TBI. We use functional connectivity (FC) to infer interactions between these networks (Friston et al., 1997). We investigate whether stopping is associated with increased FC between the rAI and the DMN and whether damage to the structural integrity of the SN leads to a change in its FC with the DMN, using psychophysiological interaction (PPI) (Friston et al., 1997). We tested the specific hypotheses that: (1) stopping would normally be accompanied by increased FC between the rAI and the DMN; (2) patients with impairments of response inhibition would fail to show this change in FC; and (3) the amount of damage to the rAI-preSMA/dACC tract would inversely correlate with FC between the $\mathrm{rAI}$ and DMN during stopping. We then replicated the analysis in a second group of TBI patients to test whether the observed effects are seen generally in situations where motor behavior needs to be changed.

\section{Materials and Methods}

\section{Patient demographics and clinical details}

TBI patient Group 1. Sixty-five patients with a history of TBI were investigated using the SST. Eight were not included in the imaging analyses because: (1) two were unable to perform the task accurately; (2) five were excluded because of distortion on the preprocessed fMRI or DTI data; and (3) one patient had an unexpected neurological abnormality on the scan. Therefore, 57 patients were included in the analyses reported here (11 females, mean age $36.7 \pm 11.5$ years, range 18-62 years). A battery of standardized neuropsychological tests designed to be sensitive to cognitive impairments commonly observed after TBI was used to compare TBI patients with an age-matched control group (Table 1). Most patients had injuries secondary to either road traffic accidents (35\%), assaults (24\%), falls $(21 \%)$, or sports injury (12\%). A further $8 \%$ of patients suffered a TBI from an unknown cause. Based on the Mayo classification system for TBI severity (Malec et al., 2007), there were 44 moderate/severe and 13 mild cases of TBI. The Mayo classification integrates the duration of loss of consciousness, length of post-traumatic amnesia (PTA), lowest recorded Glasgow Coma Scale (GCS) score in the first $24 \mathrm{~h}$, and initial neuroimaging results. GCS was recorded in 27 patients (mean $9.63 \pm$ 4.93), and average length of PTA in 49 patients was $12.05 \pm 18.99 \mathrm{~d}$. LOC was recorded in 32 patients. The exclusion criteria were as follows: neurosurgery, except for invasive intracranial pressure monitoring (one patient); history of psychiatric or neurological illness before their head injury; history of significant previous TBI; antiepileptic medication; cur- rent or previous drug or alcohol abuse; or contraindication to MRI. All patients were in the postacute/chronic phase after TBI (mean 20 months, range 2-96 months).

TBI patient Group 2. Thirty-four different patients with a history of TBI were investigated using a motor switch task. There was no overlap between patient Groups 1 and 2. Three patients were not included in the imaging analyses because: (1) two patients were unable to perform the task accurately, and (2) one was removed because of distortion on the imaging files, likely movement artifact. Therefore, the second patient group consisted of 31 patients with a history of TBI ( 10 females, mean age 37.3 years \pm 11.9 , range 20-62 years). Neuropsychological tests were performed as in Group 1 (Table 1). Most patients had injuries secondary to road traffic accidents $(48 \%)$, falls $(25 \%)$, assaults $(10 \%)$, or an incidental blow (3\%). A further $14 \%$ of patients suffered a TBI from an unknown cause. Based on the Mayo classification system for TBI severity (Malec et al., 2007), there were 24 moderate/severe and 2 mild cases of TBI, with 5 patients unknown. GCS was recorded in 8 patients (mean $10 \pm 4.75$ ), and average length of PTA for 23 patients was $9.81 \pm 23.11 \mathrm{~d}$. LOC was recorded in 14 patients. The exclusion criterion was the same as patient Group 1. All patients were in the postacute/chronic phase after TBI (mean 15 months, range 2-88 months).

Clinical imaging. Patients had standard T1 MRI to assess evidence of focal brain injury and gradient echo imaging to identify any evidence of microbleeds, a marker of diffuse axonal injury (Scheid et al., 2003). A senior consultant neuroradiologist reviewed all study MRI scans. At the time of the study, the scans of Group 1 showed the following: 11 patients had residual evidence of contusions, 12 had microbleeds (as demonstrated on gradient echo imaging), and 10 had evidence of both. In Group 2, 9 patients had residual evidence of contusions, 10 patients had microbleeds (as demonstrated on gradient echo imaging), and 5 had evidence of both. Contusions were mainly situated in the inferior parts of the frontal lobes, including the orbitofrontal cortex, and the temporal poles, in a typical lesion distribution for TBI patients (Gentry et al., 1988).

Control groups. Twenty-five controls ( 8 females, mean age $34.2 \pm 9.6$ years) were included in the SST analysis. Twenty control participants (10 females, mean age $28.5 \pm 8.7$ years) were included in the motor switch analysis. A further 30 healthy controls were used for the DTI study (16 females, mean age $37.2 \pm 8.9$ years). The Hammersmith and Queen Charlotte's and Chelsea Research ethics committee approved the study, and all the participants gave written informed consent.

Neuropsychological assessment. A detailed neuropsychological battery was used to assess cognitive function. Verbal and nonverbal reasoning ability was assessed using the Wechsler Abbreviated Scale of Intelligence Similarities and Matrix Reasoning subtests (Wechsler, 1999). Verbal Fluency Letter Fluency and Color Word Interference (Stroop test) were administered from the Delis-Kaplan Executive Function System to assess cognitive flexibility, inhibition, and set-shifting (Delis et al., 2001). The Trail Making Test (A and B) was used to further information processing speed and executive function (Reitan and Wolfson, 2004). Working memory was assessed via the Digit-Span subtest of the Wechsler Memory Scale, third edition (Wechsler, 1999). The Logical Memory I and II subtests of the Wechsler Memory Scale, third edition were included as measures of immediate and delayed verbal recall. The People test from the Doors and People test battery was used a measure of associative learning and recall (immediate and delayed) (Baddeley et al., 2003).

SST procedure. The SST was used to study response inhibition in the scanner (Sharp et al., 2010; Bonnelle et al., 2012). The SST is a two-choice reaction time task where participants are required to respond to visual stimuli using a right or left button press (see Fig. $1 A$ ). On "Go" trials, participants must respond to a left arrow by pressing the left button and a right arrow by pressing the right button. On "Stop" trials, participants must withhold their response to the arrow cues. Participants are presented by a fixation cross for $500 \mathrm{~ms}$, followed by the go stimulus for 1400 $\mathrm{ms}$. A total of $20 \%$ of the trials involved an unexpected stop signal (red dot), which was presented at a variable delay following the go signal. This is known as the stop signal delay (SSD). On $10 \%$ of the trials, the fixation cross remained on the screen. These were known as rest trials. The SST is interpreted in terms of the "horse-race" model of response inhibition (Logan et al., 1984). This model proposes that response inhibition is a 
race between an excitatory and an inhibitory process. The speed of the excitatory process determines the reaction time following the go signal. If the excitatory process is completed before the inhibitory process, then the response is executed. However, if the inhibitory process surpasses the excitatory process, the response is interrupted and therefore successfully inhibited. Consequently, the inhibition of a response depends on the relative finishing times of the two processes subsequent to the stop signal (inhibitory) and the primary go signal (excitatory).

Staircase adaptation procedure. In the first run of the SST, the SSD (delay between the presentation of the Go signal and the Stop signal) started at the mean Go reaction time (RT) of the CRT minus $200 \mathrm{~ms}$. Subsequently, the SSD was adaptively varied every two stop trials. If cumulative accuracy was $>50 \%$, the SSD was increased by $50 \mathrm{~ms}$; if $<50 \%$, the SSD was decreased by $50 \mathrm{~ms}$. A lower limit for SSD was set to $50 \mathrm{~ms}$. Therefore, a "critical" SSD could be computed for each subject per run, which represents the time delay required for the subject to succeed in withholding a response in the Stop trials for half of the time. Stop signal reaction time (SSRT) was then calculated by subtracting the critical SSD from the median Go RT for each run.

Strategic slowing down. A frequent strategy consists of slowing down on Go trials to increase the chances of successfully inhibiting the response in the case a Stop signal would appear. However, it is important for the correct estimation of the SSRT that the Go RT reflects the subjects' "real" RT (see below). We thus limited the ability of individuals to slow down on Go trials by providing negative feedback when subjects slowed their response times and passed a threshold for the speed of their Go response. Negative feedback in the form of the words "Speed up!" was presented on the screen in place of the subsequent trial each time a response was made with a reaction time above the 95th percentile of the subject's current reaction time distribution.

SSRT estimation and exclusion. The SSRT (the speed of the inhibitory process) is thought to represent the latency between the occurrence of the Stop signal and the beginning of the Stop process. Because successful response inhibition does not result in an observable response, it must be estimated (Logan et al., 1984). With the tracking procedure mentioned above, subtracting the mean SSD from the mean Go RT can derive the SSRT. For the SSRT to be accurately estimated, the Stop accuracy must be close to $50 \%$ (this is to ensure that the staircase procedure has been successful), and the strategic slowing must be within a reasonable limit. Too much slowing usually artificially lowers the SSRT estimation (Alderson et al., 2008). We thus excluded subjects whose Stop accuracy was not comprised within $40 \%-60 \%$ and subjects who had a number of negative feedback higher than 2 SDs above the group mean. With these exclusion criteria, SSRT could not be estimated on both SST runs in 3 controls and 11 patients. The analyses involving this measure are thus reported for a sample of 22 controls and 46 patients.

Motor switch paradigm procedure. To investigate mental processes involved in reconfiguring task-sets, we instructed our participants to consistently switch between tasks and examined the behavioral and neural correlates of this switching. We implemented a task-switching paradigm (see Fig. $1 B$ ). This was a two-choice task-switching paradigm, which requires reconfiguration of mental resources to execute an appropriate motor response (Monsell, 2003) based on a Switch cue (Sudevan and Taylor, 1987). The paradigm required participants to classify target stimuli as either blue or red. Initially, participants were required to respond to blue targets with their left hand and red targets with their right hand. This part of the task was performed for a variable period of time. Trials that followed one another without change in this motor response mapping were classed as Go trials (stimulus duration $=2000 \mathrm{~ms}$ ). At unpredictable intervals, participants were presented with a Switch cue $(1000 \mathrm{~ms})$, which preceded a Switch trial $(2000 \mathrm{~ms})$. This Switch cue (Sudevan and Taylor, 1987) informed the participant to switch their motor response mapping on subsequent trials. Therefore, participants were required to respond to blue targets with their right hand and red targets with their left hand. The paradigm contained 162 trials, with $20 \%$ of the trials constituting Switch trials. The interstimulus interval was $3000 \mathrm{~ms}$ with each trial $(2000 \mathrm{~ms})$ broken up by a fixation cross $(1000 \mathrm{~ms})$. Participants were trained on the paradigm before scanning over a period of 62 trials. Participants were also asked to recall the motor mapping rules before enter- ing the scanner. To reduce the memory load, we presented participants with the name of the current rule above target stimuli. We recorded participants' accuracy and RT over the entire run. RT was calculated by subtracting the time to respond to the target stimuli from the onset of the target stimuli.

MRI image acquisition. MRI data were obtained using a Philips Intera 3.0-T MRI scanner using Nova Dual gradients, a phased-array head coil, and sensitivity encoding with an undersampling factor of 2 . fMRI images were obtained using a T2*-weighted gradient-echo echoplanar imaging sequence with whole-brain coverage (repetition time/echo time, 2000/30 ms; 31 ascending slices with thickness $3.25 \mathrm{~mm}$, gap $0.75 \mathrm{~mm}$, voxel size $2.5 \times 2.5 \times 5 \mathrm{~mm}$, flip angle $90^{\circ}$, field of view $280 \times 220 \times 123 \mathrm{~mm}$, matrix $112 \times 87)$. Quadratic shim gradients were used to correct for magnetic field inhomogeneities within the brain. T1-weighted wholebrain structural images were also obtained in all subjects. Paradigms were programmed using MATLAB Psychophysics toolbox (Psychtoolbox-3, MathWorks; www.psychtoolbox.org) and stimuli presented through an IFIS-SA system (In Vivo). Responses were recorded through a fiberoptic response box (Nordicneurolab), interfaced with the stimulus presentation PC running MATLAB. Participants had two imaging sessions: one session consisted of structural brain imaging including DTI and another of task fMRI.

Diffusion-weighted volumes with gradients applied in 64 noncollinear directions were collected. The following parameters were used: 73 contiguous slices, slice thickness $=2 \mathrm{~mm}$, FOV $224 \mathrm{~mm}$, matrix $128 \times 128$ (voxel size $=1.75 \times 1.75 \times 2 \mathrm{~mm}^{3}$ ), $\mathrm{b}$ value $=1000$, and four images with no diffusion weighting $\left(b=0 \mathrm{~s} / \mathrm{mm}^{2}\right)$. Diffusion-weighted images were registered to the $\mathrm{b}=0$ image by affine transformations to minimize distortion resulting from motion and eddy currents and then brainextracted by using BET (Smith, 2002), part of the FSL image processing toolbox (Smith et al., 2004). Using FDT in FSL, we generated voxel wise fractional anisotropy (FA) maps.

\section{Neuroimaging analysis}

A high level overview of the methodology used is presented in Figure 1 and further explained in the subsections below. Briefly, our dataset provided us with behavioral, functional, and structural imaging data. We investigated regional changes in brain activity in response to stopping and switching (see Regional brain activation and ROIs) as well as changes in the interaction between brain regions (see FC analysis). Furthermore, we performed tractography on an independent group of healthy volunteers to define a rAI-preSMA/dACC mask, and we investigated the structural integrity of this tract in our patients (see Structural white matter tractography analysis). Together, this analysis pipeline allowed us to investigate how damage to the rAI-preSMA/dACC white matter tract influences interactions between the SN and DMN.

Regional brain activation. All the functional data were analyzed using a platform inside FSL version 4.1.9 [FMRIB Software Library (Smith et al., 2004) known as FEAT (FMRI Expert Analysis Tool) version 5.98]. The following prestatistics processing was applied; motion correction using MCFLIRT (Jenkinson et al., 2002); nonbrain removal using BET (Smith, 2002); spatial smoothing using a Gaussian kernel of FWHM 5 mm; grand-mean intensity normalization of the entire $4 \mathrm{D}$ dataset by a single multiplicative factor; high pass temporal filtering (Gaussian-weighted least-squares straight line fitting, with $\sigma=50.0 \mathrm{~s}$ ). Mixed effects analyses of group effects were performed using the FMRIB local analysis of Mixed Effects. The final $\mathrm{Z}$ statistical images were thresholded using a Gaussian random field-based cluster inference with a height determined by a threshold of $Z>2.3$ and a (corrected) cluster significance threshold of $p=0.05$. fMRI data were analyzed using voxelwise time-series analysis within the framework of the general linear model (GLM) (Beckmann et al., 2003). A design matrix was generated with a synthetic hemodynamic response function and its first temporal derivative. Several types of events were distinguished for the SST data. These included: Go correct (Go), Stop correct (StC), Stop incorrect (StI), and Rest. Go incorrect trials were not included as there were too few to model accurately. To account for variation in the SSD across runs, we modeled events by using the timing of the SSD as the regressor for each trial. The following subject specific- and run-specific contrasts were generated: StC versus Go and Go versus Rest. For the motor switch paradigm, the 
following events were generated: Go correct (Go), Switch correct (SwC), Errors (Er), and no response (NoR), and the following contrasts were generated:SwC > Go and Go $>$ SwC.

ROIs. For the FC analysis, we investigated activity in three ROIs previously defined by Bonnelle et al. (2012). These were centered on peaks of activation for the contrasts of correct Stop with Go trials. $10 \mathrm{~mm}$ radius spheres from the $\mathrm{rAI}(x=36, y=24, z=-6)$ and the $\operatorname{dACC}(x=0, y=$ $22, z=46)$ were used, corresponding to two major nodes of the SN. In Bonnelle et al. (2012), these ROIs were used as the seed points to define the tract connecting the rAI and AACC; this tract was the primary focus of our current analysis. We also defined a right inferior frontal gyrus (rIFG, $x=44, y=18, z=16$ ) ROI, to test whether connectivity changes are specific to the salience network (Sharp et al., 2010). These three masks were applied to both Switching and Stopping data.

FC analysis. The first stage in the FC analysis involved extracting time courses in individual subjects from the three frontal ROIs, as well as the DMN. We used the first stage of the dual regression pipeline to extract time courses from the DMN and our three frontal ROIs in individual subjects (Filippini et al., 2009; Zuo et al., 2010; Leech et al., 2011), which were then used in the psychophysiological interaction analysis (O'Reilly et al., 2012) (described below). To provide an unbiased estimate of DMN activity, we used a map for this network based on the independent component analysis of fMRI data from Smith et al. (2009). Dual regression involves back-projecting (spatially regressing) each ICA component into individual subject's $4 \mathrm{D}$ functional data to derive a time course from each subject for the DMN. This time course is the subject-specific signal fluctuation corresponding to each group-level independent component. In addition to the DMN component, we also included 14 noise components, also taken from the Smith analysis. These provide group-level estimates of various sources of noise in the data, including motion, cerebrospinal fluid, and white matter signals. The dual regression approach has a number of advantages over the use of seed voxel-based approaches and has been used to probe behavioral and pathology-related differences in multiple populations (Damoiseaux et al., 2008; Filippini et al., 2009). It provides a data-driven way of extracting time-series that best fit particular regions or networks. The procedure also controls for the potential confounds, such as motion that may be present in the data. This procedure was used to define a time course from the DMN and frontal ROIs for each run of the SST dataset for each subject, and the Motor Switch paradigm for each subject.

A GLM was then used to perform the PPI analysis to examine the change of FC during Stop versus Go trials and Switch versus Go trials in a standard way (Friston et al., 1997; O’Reilly et al., 2012). A task-specific change in FC suggests a "change in the exchange of information" between regions (O'Reilly et al., 2012). This approach allows us to measure task related changes in FC and separate these from changes in regional brain activation. Following O'Reilly et al. (2012), the interaction time course is an element-by-element product of the mean-centered task time course and demeaned seed ROI time course. This GLM was calculated for each subject, including the subject-specific DMN time course as the dependent variable. The independent variables were as follows: (1) the time course of each frontal ROI (assessing FC unrelated to task); (2) the Stop/ Switch, Go, and error time courses (modeling the main effects of task events); (3) confounding variables consisting of motion parameters and individualized time courses of all 14 noise components from the dual regression, such as the subject-specific time courses for motion components included in the dual regression (Smith et al., 2009); (4) the interaction time courses between Stop and the ROI (Stop $\times$ ROI) and between Go and the ROI (Go $\times$ ROI); and (5) a constant. The GLM resulted in parameter estimates (PEs) for each of the independent variables, and the PEs for the interaction terms were contrasted (i.e., Stop interaction-Go interaction). A positive contrast suggests that change in the relationship between DMN and the ROI resulting from the task event is stronger during Stop (or Switch) events than during Go events.

Structural white matter tractography analysis. Building on our previous results (Bonnelle et al., 2012), we were interested in investigating the tract connecting the rAI-preSMA/dACC. The method for defining this tract in an unbiased way has been described previously, and the tract location for all subjects in Group 1 is contained in Bonnelle et al. (2012, Supplementary Material). In summary, we initially defined a group average for this tract by performing individual tractography on an independent group of 10 young normal controls ( 6 males, mean age $23 \pm 2.5$ years) using standard techniques (Hua et al., 2008; Squarcina et al., 2012). Tracts were generated between $10 \mathrm{~mm}$ radius spherical regions of interest placed on the peak activation or deactivation during the Stop versus Go contrast using probabilistic tractography in FSL. FA maps were nonlinearly warped and registered to the $1 \mathrm{~mm}$ FMRIB MNI FA template, by using FSL FNIRT, and the obtained transformations were used to bring the individual tractography outputs to the standard space. The projected tracts were then averaged across the 10 subjects. For the resulting map, a conservative threshold corresponding to $5 \%$ of voxels with highest connectivity values was used. Tractography was performed from the rAI to dACC and from dACC to rAI. The two resulting thresholded tracts were then averaged and binarized.

This rAI-dACC tract was used as a mask for the ROI analysis of white matter integrity in TBI patients and in a group of 30 age-matched controls distinct from the one used to generate the tracts. This tract was projected into each individuals DTI space by using the inverse of the nonlinear transformation used to align the subject-space FA maps to the MNI template. To reduce the possibility of sampling nonwhite matter regions, the transformed tracts were constrained within a skeleton of white matter tracts mask derived from TBSS (Smith et al., 2006). This allows sampling of only the core of the tract while excluding peripheral parts of the fiber tract that show pronounced interindividual variability. The obtained maps were binarized and applied to the FA maps to obtain one mean FA value per tract and per subject. Mean FA values were thus calculated from the area of overlap between the whole white matter skeleton and the mask of the particular tract in individual space. We then used linear regression to derive FA values corrected for any effects of age in the analyses reported. Furthermore, to investigate the specificity of this rAI-preSMA/dACC tract, we analyzed four other tracts that connected regions that were either activated or deactivated during response inhibition, which were previously described by Bonnelle et al. (2012). Briefly, these tracts include connections between (1) the precu/posterior cingulate cortex (PCC) and the ventromedial prefrontal cortex, (2) the rIFG and the preSMA, (3) the rIFG and the right temporoparietal junction, and (4) the right frontal eye fields and right intraparietal sulcus.

\section{Results \\ Stopping: TBI Group 1}

Behavior and neuropsychological assessment

Our first analysis involved 57 TBI patients ( 11 females, age $36.7 \pm$ 11.5 years) and 25 healthy controls who performed the SST. Here, sudden increases in motor control are studied on $20 \%$ of the trials where subjects attempt to stop a motor action in response to an unexpected stop signal (Fig. 1A). We have previously reported the behavioral results for this patient group (Bonnelle et al., 2012). In brief, the TBI patients showed an expected pattern of neuropsychological impairment, with evidence of slow information processing speed, impaired inhibition, and reduced cognitive flexibility (Table 1). The SSRT, a measure of inhibitory processing, was significantly longer in patients than age-matched controls indicating an impairment of response inhibition $(t=2.01$, $\mathrm{df}=66, p=0.014)$. Despite these impairments, both patients and controls were able to perform other aspects of the SST well, with $\sim 50 \%$ Stop accuracy and $>95 \%$ Go accuracy (Table 2).

\section{FC analysis}

To investigate the interaction between the $\mathrm{SN}$ and $\mathrm{DMN}$, we focused on two core nodes of the SN, the rAI and the dACC, as well as the rIFG, a region adjacent to the rAI. ROIs were defined on the basis of patterns of activity observed during stopping. We have previously reported the following: (1) the rAI and dACC show increased activity during stopping; (2) the DMN, including the PCC, normally shows reduced activity during stopping; and (3) TBI results in a failure of DMN deactivation, which is predicted by the amount of structural damage to the white matter 


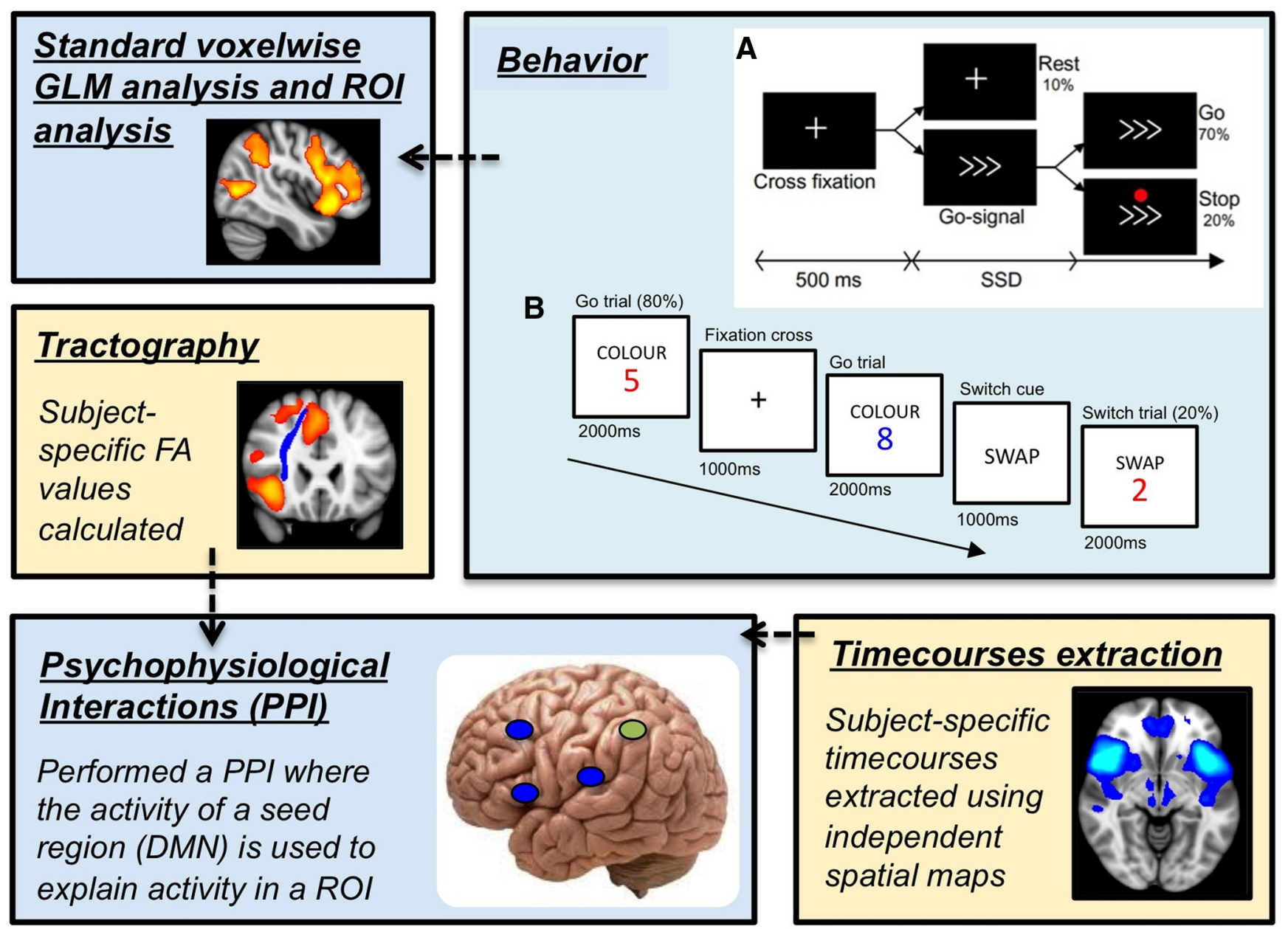

Figure 1. Methodology pipeline describing the techniques used in our analyses. The initial fMRI data were analyzed using a univariate approach to generate contrasts between key regressors (such as Stop $>$ Go and Switch $>$ Go). We then implemented the first stage of dual regression to extract subject-specific time courses for the DMN. The DMN spatial map came from an independent dataset defined by Smith et al. (2009). These time courses were then implemented in our psychophysiological interaction, where we calculated GLMs for each subject. This GLM included the DMN time course as our dependent variable. The independent variables included a constant, the time course of our seed ROI (e.g., the rAl), task time courses, and interaction time courses. The GLM generated parameter estimates for our independent variables, where we contrasted the task interactions (e.g., Stop $\times$ ROl interaction-Go $\times$ Rol interaction parameter estimates) to replicate our contrasts at the univariate level (e.g., Stop $>$ Go). $\boldsymbol{A}$, Schematic overview of the stop signal task. $\boldsymbol{B}$, Schematic overview of the motor task switching paradigm.

Table 2. Behavioral results ${ }^{a}$

\begin{tabular}{lcc}
\hline & Control group 1 & TBl group 1 \\
\hline SST & & \\
Median RT (ms) & $443 \pm 106$ & $483 \pm 116$ \\
Go \% accuracy & $98.4 \pm 1.5$ & $95.5 \pm 4.8^{*}$ \\
Stop \% accuracy & $49.5 \pm 2.2$ & $49.6 \pm 2.6$ \\
Negative feedback & $13 \pm 8$ & $12 \pm 9$ \\
IIV & $0.181 \pm 0.037$ & $0.188 \pm 0.054$ \\
SSRT (ms) & $238 \pm 32$ & $268 \pm 67^{*}$ \\
& Control group 2 & TBI group 2 \\
\hline Motor switch paradigm & & \\
Switch trial RT (ms) & $745 \pm 100$ & $898 \pm 100^{* *}$ \\
Go trial RT (ms) & $619 \pm 100$ & $807 \pm 100^{* *}$ \\
Switch cost (ms) & $127 \pm 100$ & $97 \pm 100$ \\
Switch trial \% accuracy & $92.3 \pm 7.8$ & $84.9 \pm 14.4^{*}$ \\
Go trial \% accuracy & $97 \pm 2.9$ & $90.8 \pm 13.5^{*}$ \\
\hline
\end{tabular}

${ }^{a}$ Behavioral results for the SST and motor switch paradigm. Data are mean \pm SD.

${ }^{*} p<0.05,{ }^{* *} p<0.005$, significant differences between patients and controls.

tract connecting the rAI and the dACC (Sharp et al., 2010; Bonnelle et al., 2012) (Fig. 2). The specific nodes we studied in the PPI analysis showed regional activity changes during stopping consistent with this overall pattern (Fig. 3A).
Hypothesis 1: stopping is normally accompanied by increased FC between the rAI and the DMN

FC between the DMN and the SN changed during stopping. In healthy subjects, a one-way ANOVA of the PPI between the three ROIs and the DMN showed a main effect of region $(F=5.5 \mathrm{df}=$ 1.5,39, $p<0.02$; Huyn-Feldt correction applied; Fig. $4 A$ ). Planned contrasts between the rAI PPI and each of the other two ROIs showed that the FC with the DMN during stopping was significantly higher for the rAI than either the dACC or $\operatorname{rIFG}(F>$ $6, \mathrm{df}=1,24, p<0.02$ in each case). One-sample $t$ tests show that the increase in FC during stopping between the DMN and the rAI was significantly greater than zero $(t=4.2, \mathrm{df}=24, p<0.001)$ but was not significantly above zero for the dACC or rIFG. It is noteworthy that the increase in FC between the SN and DMN occurred in the context of distinct patterns of relative activation change: an increase in the rAI, but a decrease in the DMN.

Hypothesis 2: patients with impaired performance on the SST fail to show increased FC during stopping

Cognitive impairment on the SST in TBI patients was associated with abnormalities of FC (Fig. $4 A$ ). A $2 \times 3$ Group $\times$ Region ANOVA, adding in the patient group, showed a significant region $\times$ group interaction $(F=5.95, \mathrm{df}=2,160, p=0.003)$. 


\section{A Stopping}

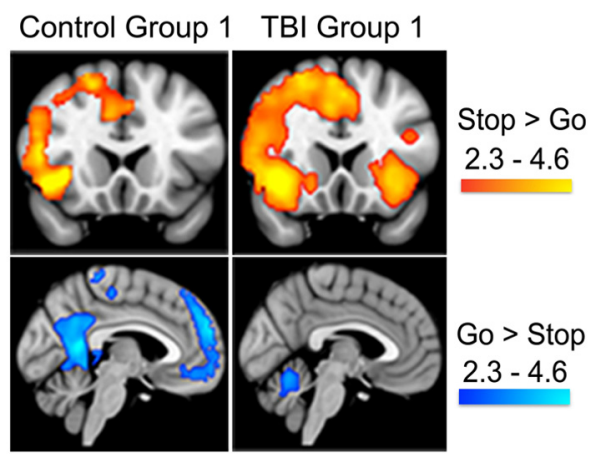

\section{B Switching}

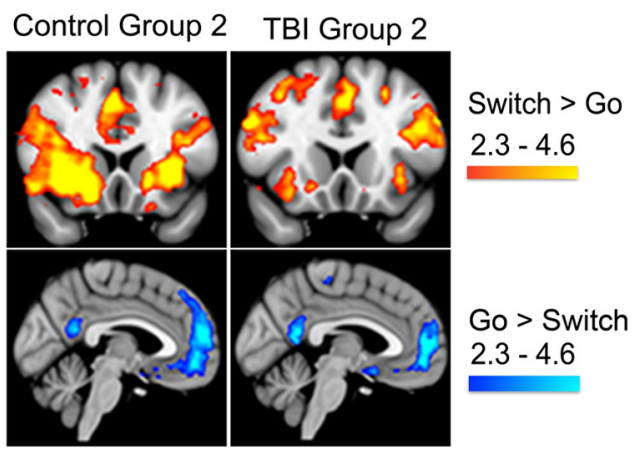

Figure 2. Brain activation patterns during Stopping and Switching. $\boldsymbol{A}$, Overlay of brain activation associated with correct $S$ top ( $\mathrm{StC}$ ) versus $\mathrm{Go}$ trials for controls and patients. $\boldsymbol{B}$, Overlay of brain activation associated with correct Switch (SwC) versus Go trials for controls and patients. Results are superimposed on the MNI-152 $\mathrm{T} 11 \mathrm{~mm}$ brain template. Cluster corrected $Z=2.3$, $p<0.05$.

Planned contrasts comparing FC of the DMN with the rAI and each of the other two ROIs in interaction with the Group factor showed that the increase in FC for the rAI compared with either of the other ROIs was significantly greater in the controls than in the patients $(F>7.8, \mathrm{df}=1,80, P<0.006$ in each case). Pairwise $t$ tests (comparing controls and patients) revealed a significant group difference in the PPI between the rAI and DMN $(t=3.72$, $\mathrm{df}=28, p=0.001$; corrected for inhomogeneity of variance between groups), but there were no group differences in the other regions ( $t<0.91, p>0.35$ in each case) (Fig. $4 A)$. In the patients, there was no significant PPI in any of the three regions tested and no regional differences in PPI magnitude.

Hypothesis 3: damage to connections of the SN causes a failure of normal functional interactions with the DMN

We next investigated in patients whether the integrity of the SN tract connecting the rAI to the pre-SMA/dACC correlated with abnormalities of FC between the SN and DMN (Fig. $5 A, B$ ). FA in this tract in patients was significantly lower compared with controls $(t=3.62, \mathrm{df}=85, p=0.001)$. We found a significant positive correlation between $\mathrm{SN}$ tract integrity and the strength of PPI between the DMN and $\mathrm{rAI}(r=0.4, p=0.003)$. Patients with more damage to this tract (i.e., lower fractional anisotropy) showed a weaker PPI (i.e., FC increased less during stopping) (Fig. 5A). There was no significant correlation between the same white matter tract and the PPI between the DMN and the dACC (Fig. $5 B$ ) or rIFG. There was also no significant correlation between any FC measurement with the DMN and the other four
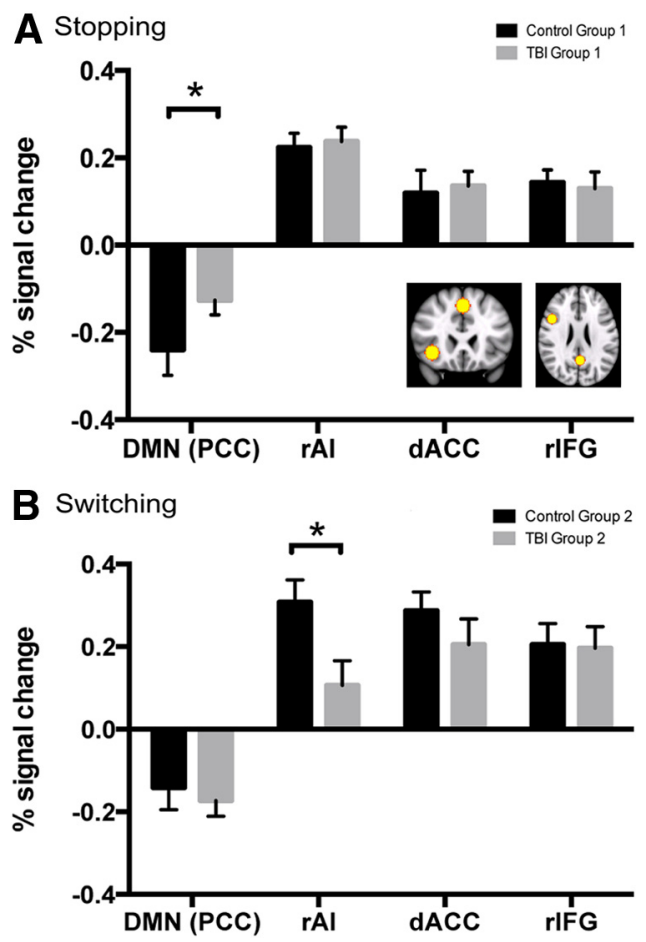

Figure 3. Regional brain activation during Stopping and Switching. BOLD percentage signal change during correct Stop trials versus Go trials in the PCC, the rAI, the $\mathrm{AACC}$, and the rIFG in Stopping $(\boldsymbol{A})$ and Switching $(\boldsymbol{B})$. Inset, Spatial maps showing ROI positions superimposed on the MNI $1521 \mathrm{~mm}$ brain template. ${ }^{*} p<0.05$.

white matter tracts studied, which connects regional activity during stopping (Bonnelle et al., 2012).

SN disconnection, activation, and behavior on the SST

There was also a correlation between the integrity of the rAI-preSMA/dACC tract and strength of activation of the rAI during stopping in the patients. Damage to the tract was associated with less activation of the rAI during stopping $(r=0.25, p=0.02)$. In contrast, there were no significant correlations between the integrity of the white matter tract and either dACC or rIFG activation during stopping. In the patient group, greater rAI activity during stopping was also associated with more efficient response inhibition (lower SSRT) $(r=-0.28, p=0.016)$. This relationship was again not observed in the dACC and the rIFG. There was no significant correlation between SSRT and the PPI between the DMN and any of the nodes studied. We found no significant relationship between FA and behavior in the four other tracts studied. Furthermore, we investigated whether injury severity (based on the Mayo classification) was related to our experimental measures. For Group 1, we conducted independent samples $t$ tests on tract FA, behavior, and PPI and found no significant differences between patients with mild (probable) and moderate to severe injuries.

\section{Switching: TBI Group 2}

Behavior and neuropsychological assessment

To test whether the observed effects were reproducible and whether they generalized to other types of motor control, we investigated a second completely separate group of TBI patients and healthy controls who performed a motor switch task. Thirtyone patients with a history of TBI ( 10 females, mean age $37.3 \pm$ 11.9 years) and 20 controls (10 females, mean age $28.5 \pm 8.7$ years) were scanned performing a motor switch task. Subjects 

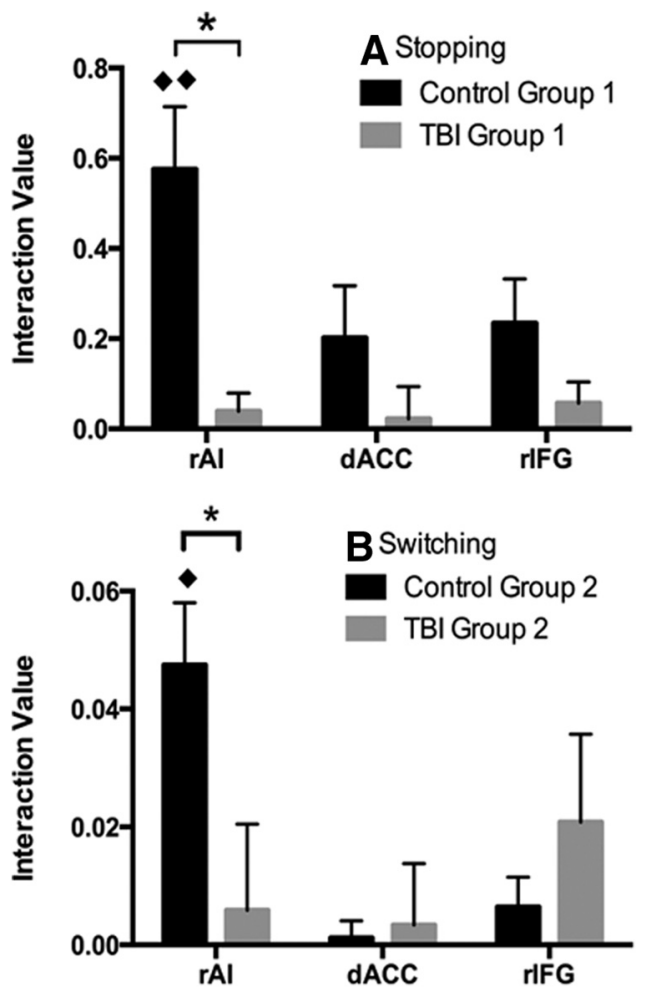

Figure 4. Psychophysiological interaction analysis during Stopping and Switching. Bar charts represent the strength of the psychophysiological interaction produced by Stopping $(\boldsymbol{A})$ and Switching $(\boldsymbol{B})$ with the DMN and our three ROls in controls (black) and patients (gray). $\diamond p<0.001 . \diamond p<0.005$. ${ }^{*} p<0.005$.

made motor responses with either the right or left hand on the basis of the color of a visual cue (Fig. $1 B$ ). As with the SST, subjects had to change their normal motor action on $20 \%$ of the trials, by changing the hand with which they made a response on Switch trials (e.g., from blue requiring a response with the right hand, to blue requiring a response with the left hand).

Again, this patient group showed a predictable pattern of neuropsychological impairment with evidence of slow information processing speed, impaired inhibition, reduced executive functions, and working memory capacity (Table 1). They also showed impairments on task performance in the scanner. Accuracy on Go trials for both groups was generally high $(>90 \%)$, although patients were slightly less accurate than controls on both Go $(t=$ $2.08, \mathrm{df}=50, p=0.02)$, and Switch trials $(t=2.03, \mathrm{df}=50, p=$ $0.02)$. Patients were also slower to respond to Switch trials than controls $(t=-4.17, \mathrm{df}=50, p<0.005)$, as well as Go trials $(t=$ $-5.41, \mathrm{df}=50, p<0.005)$ (Table 2).

\section{Regional changes in neural activity during switching}

Brain activity during switching was similar to previous studies in both patients and controls (Fig. 2B) (e.g., Dosenbach et al., 2006; Kim et al., 2011; Leunissen et al., 2013). Switch compared with Go trials showed increased activity in the anterior cingulate cortex/preSMA, bilateral inferior and middle frontal gyri, frontal operculum cortex, and insular cortex. More posteriorly, activation was observed within the precuneus cortex, lingual gyrus, supramarginal gyrus, and intraparietal sulcus and the lateral occipital cortices. Decreased activity was observed in the ventromedial prefrontal cortex, superior frontal gyrus, and the PCC (Fig. $2 B)$. The direct contrast of control and patient groups showed reduced activity in patients within the rAI, frontal operculum cortex, precentral gyrus, frontal orbital cortex, and paracingulate gyrus.

\section{FC analysis}

Similar PPI results to stopping were observed in switching: Hypothesis 1 (Fig. 4B): For controls, a significant PPI was seen between the rAI and DMN for switching $(t=5.52$, df $=19, p<$ $0.005)$. This was not present for either the dACC or the rIFG. Hypothesis 2 (Fig. 4B): An ANOVA showed a significant region $\times$ group interaction for the PPI $(F=4.85, \mathrm{df}=1,48, p<$ $0.005)$, the result of a significantly weaker PPI in patients than controls between the rAI and DMN $(t=3.17, \mathrm{df}=48, p=0.003)$. In the patients, there was again no significant PPI in any of the three regions tested and no regional differences in PPI magnitude. Hypothesis 3 (Fig. 5C,D): The white matter tract connecting the rAI to the pre-SMA/dACC showed significantly lower FA in patients compared with our healthy control group $(t=-6.24$, $\mathrm{df}=59, p<0.001)$. Within patients, the integrity of the rAIpreSMA/dACC tract was again positively correlated with the strength of PPI between the rAI and the DMN $(r=0.4, p=0.03)$ (Fig. $5 C$ ), such that greater damage to this tract was associated with reduced strength of PPI. There was no significant relationship observed between either the dACC (Fig. 5D) or rIFG and the DMN.

SN disconnection, activation, and behavior on the motor switch In contrast to stopping, we found no relationship between the integrity of the rAI-preSMA/dACC tract and the amount of rAI activation during switching in either participant group, or any relationship between rAI activation and task switching in the scanner. However, a standard neuropsychological measure of task switching (Trail Making A-B) measured outside the scanner was negatively correlated with $\mathrm{rAI}$ activation in the patients $(r=$ $-0.35, p=0.01)$, such that less activation was associated with greater switch cost.

\section{FC results remains significant after removing patients with} focal lesions

To exclude an artifactual effect of focal lesions on our main findings, we repeated our PPI analyses, excluding patients with focal cortical lesions in both stopping and switching groups. In patient Group 1, after removing 21 patients with focal lesions, there remained a significant difference between patients and controls in their PPI between the DMN and the rAI $(t=3.4$, df $=59, p=$ 0.001). Furthermore, the correlation between the PPI and the rAI-preSMA/dACC tract also remained significant $(r=0.4, p=$ 0.02 ). In patient Group 2, the PPI between the rAI and DMN remained significant even after we removed 13 patients with focal lesions $(t=3.08, \mathrm{df}=32, p=0.004)$, and the correlation between the mean FA of the rAI-preSMA/dACC tract and PPI between DMN and rAI became stronger without patients with focal lesions $(r=0.6, p=0.02)$.

\section{Analysis of motion}

Differences in FC were not the result of within-scanner motion differences between the groups. We corrected for motion in a number of ways. First, mean relative root mean squared framewise displacement, which was derived from the six motion parameters, was calculated from each subject's imaging data as part of our motion correction analysis. We then performed an independent samples $t$ test for the six motion parameters as well as the average of the six. We found no significant differences between patients and controls. Furthermore, to test any differences at the PPI level, we added the motion parameters into our multiple 
A-B: Stopping

A rAl *

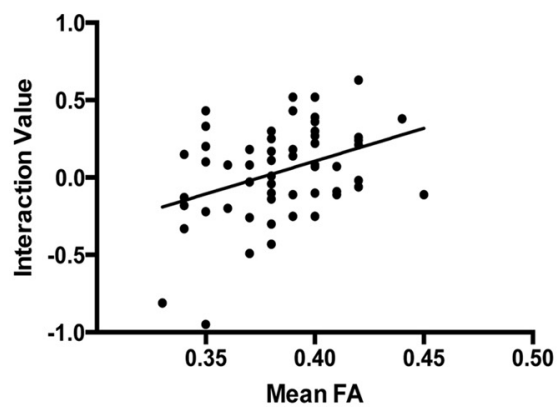

C-D: Switching

C rAl *

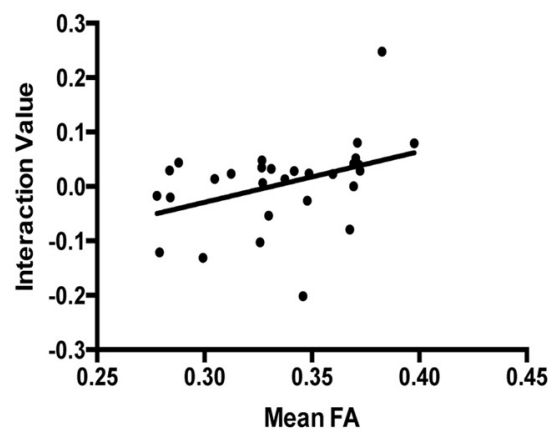

B dACC

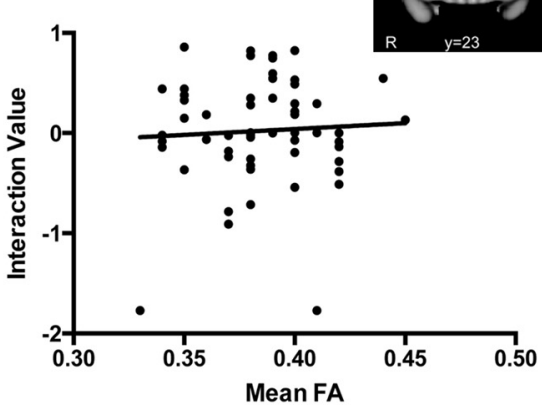

D $\mathrm{dACC}$

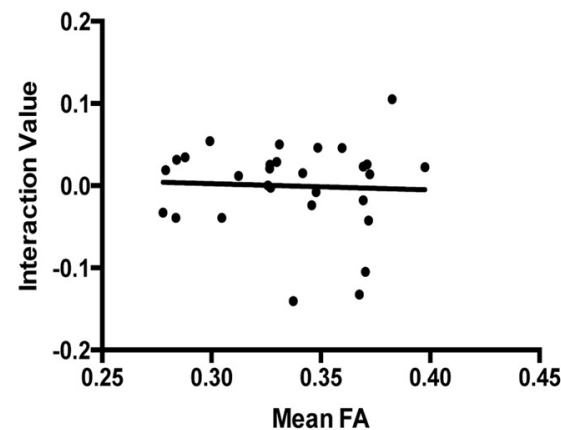

Figure 5. The relationship between disconnection of the $\mathrm{SN}$ and network interaction. Mean FA of the rAl-preSMA/dACC tract (inset) in patients plotted against: the strength of the psychophysiological interaction for Stopping between the DMN and the rAI $(\boldsymbol{A})$, the dACC $(\boldsymbol{B})$, and for Switching between the DMN and the rAl $(\boldsymbol{C})$, and the $\mathrm{dACC}(\boldsymbol{D}) .{ }^{*} p<0.05$.

regression model, alongside the task variables. This provided us with six subject-specific motion parameter regression coefficients, which we averaged, and then performed an independent samples $t$ test between groups. Again, we found no significant difference.

\section{Discussion}

Activity across brain networks must be coordinated during rapid changes in behavior. It is proposed that the SN plays a key role in coordinating activity by causally influencing the DMN (Menon and Uddin, 2010). If this occurs, damage to the $\mathrm{SN}$ should disrupt this interaction, particularly when cognitive control needs to be engaged. Here, we investigate interactions between the $\mathrm{SN}$ and $\mathrm{DMN}$ in the context of motor control by studying the impact of damage to the SN after TBI. We have previously shown that damage produced by TBI to the white matter tract connecting the rAI and dACC/pre-SMA predicts a failure to suppress DMN activity normally during stopping (Bonnelle et al., 2012). We extend this work by showing that: (1) FC between the SN and the DMN normally increases when motor responses are rapidly inhibited or changed; (2) this transient increase in FC is reduced in patients with impairments of motor control following TBI; and (3) the amount of posttraumatic damage to structural connections within the SN correlates with the extent of breakdown of this functional network interaction. We replicate the results across two separate groups of patients and show similar relationships when actions are either switched to an alternative or stopped altogether.

This provides evidence that the $\mathrm{SN}$ is required for efficient control of DMN activity when external events require rapid be- havioral response. In particular, it supports a role for the rAI in switching activity in other networks, including the DMN (Menon and Uddin, 2010). One other source of evidence for a direct influence of the rAI on DMN activity comes from fMRI studies using Granger causality analysis to infer effective (i.e., causal) connectivity (Sridharan et al., 2008; Chiong et al., 2013). Sridharan et al. (2008) observed that the rAI exerts a causal influence on the activity of the DMN across a range of behaviors and that the region had high causal outflow connections and low causal inflow connections (Sridharan et al., 2008). Using the same technique, Chiong et al. (2013) studied healthy subjects and patients with behavioral variant frontotemporal dementia, who exhibit abnormalities of SN function (Seeley et al., 2009). Subjects performed a moral reasoning task, which is impaired in these patients. A strong causal influence from the rAI to PCC was observed in healthy controls, which broke down in patients with impairments of moral reasoning, providing evidence for a causal influence of the SN on the DMN that is vulnerable to pathology within the SN (Chiong et al., 2013). However, doubt has been raised about the accuracy of Granger causality analysis when applied to fMRI data because "lag-based" methods of inferring causality may be compromised by the poor temporal resolution and auto-correlation of fMRI (Smith et al., 2011). In addition, recent work using TMS to stimulate or inhibit an anterior node of the SN failed to show evidence of causal influence of the network on the DMN, despite stimulation of an adjacent region within the central executive network modulating activity within the DMN (Chen et al., 2013). Therefore, convergent evidence for the causal influence of the SN over the DMN remains important.

The anticorrelation of activity between the SN and DMN reflects their distinct but coupled cognitive functions. The DMN shows high activity when attention is directed internally, such as during memory retrieval or when subjects thoughts are not relatively unconstrained e.g., during "resting" state scanning (Raichle et al., 2001). When attention is focused externally, activity within the DMN normally shows a load-dependent reduction as cognitive demands increase (Singh et al., 2011). Failures of DMN deactivation are associated with lapses of attention in healthy adults (Weissman et al., 2006) and are observed across many diseases (Leech and Sharp, 2014). When attention is externally focused, DMN activity is usually anticorrelated with that of the $\mathrm{SN}$, and greater anticorrelation between the $\mathrm{SN}$ and DMN is associated with more efficient cognitive control (Kelly et al., 2008). This suggests that the coupling of the two networks influences attentional focus and that controlling the balance of activity in the two networks is an important mechanism for cognitive control.

The SN typically shows increased activity in situations where attention needs to be directed externally (e.g., when actions need to be unexpectedly cancelled or changed) (Menon and Uddin, 
2010; Bonnelle et al., 2012). These situations often require a rapid response and are characterized by increased autonomic and emotional activity, in addition to changes in motor control. The rapid coordination between the DMN and the SN can perhaps be viewed as similar to a "flight or fight" response, which involves the rapid allocation of resources toward potential external threats. In this situation, attention is focused on changes in the environment, internal mental activity is rapidly curtailed, and motor control systems engaged. The robust functional link between the rAI and PCC can be seen as the neural substrate for the inhibition of internally directed cognitive activity, during the initiation of rapid changes in behavior.

The microscopic structure of the SN may be specialized for generating a rapid "circuit breaking" signal. The insular cortex and the dACC contain von Economo neurons (von Economo and Koskinas, 1925). These large bipolar projection neurons are predominantly found in the right hemisphere and are a phylogenetically recent specialization in hominoid evolution, found in humans and great apes but not other primates (Allman et al., 2010). Their large size and simple dendritic structure spanning cortical layers suggest a specialization for integrating cortical signals and rapidly communicating with remote brain regions. The "flight or fight" response puts a premium on the capacity to respond quickly to a rapidly changing environment. Therefore, the SN may have evolved to rapidly integrate information from a variety of sources to signal potentially relevant changes in the environment critical for initiating fast adaptive behavioral responses.

In our results, it is notable that an increase in FC between the rAI and PCC is accompanied by distinct local changes in brain activity (i.e., a relative increase in the $\mathrm{SN}$ and a decrease in the DMN). This is not unexpected, and it illustrates how increased "communication" between brain regions can occur at the same time as a relative reduction in the activity in one or both of the connected regions. In the case of the PCC, we have previously shown how subregions can show distinct patterns of FC change during an attentionally demanding task while at the same time the whole region shows a reduction in activity relative to baseline (Leech et al., 2012). The pattern of results we report is in keeping with a model where increased cognitive control is accompanied by increased FC between the rAI and the PCC, which through interactions between the dorsal and ventral PCC produces an overall reduction of activity and FC within the core nodes of the DMN.

Our study has a number of potential limitations. We performed a very focused analysis of FC to test a specific hypothesis, which was motivated by the specific relationship between SN integrity and DMN function (Bonnelle et al., 2012). We cannot exclude the possibility that damage elsewhere in the brain contributes to the impairment of DMN control, although the lack of a correlation between tract structure and FC in any other tract studied provides evidence against this. Future work could usefully expand the analysis in various ways. Other brain regions are undoubtedly involved in cognitive control, and so additional nodes in the network assessed should be assessed in more complex models of network interactions. We used a simple analysis of FC to maximize our power to detect relationships between brain structure and function that can be subtle. However, multivariate approaches to FC analysis could provide important additional information. Additionally, we were unable to comment on the causality of interactions between the SN and the DMN using our psychophysiological interaction technique, and this limitation might be addressed using techniques, such as dynamic causal modeling (Friston et al., 1997).
In conclusion, we show that coupling between the rAI and $\mathrm{DMN}$ increases with cognitive control and that damage within the SN impairs this dynamic network interaction. This provides compelling evidence for a model of cognitive control where the rAI signals the attentional capture of salient stimuli and interacts with the DMN to produce a reduction of its activity when attention is externally focused.

\section{References}

Alderson RM, Rapport MD, Sarver DE, Kofler MJ (2008) ADHD and behavioral inhibition: a re-examination of the stop signal task. J Abnorm Child Psychol 36:989-998. CrossRef Medline

Allman JM, Tetreault NA, Hakeem AY, Manaye KF, Semendeferi K, Erwin JM, Park S, Goubert V, Hof PR (2010) The von Economo neurons in frontoinsular and anterior cingulate cortex in great apes and humans. Brain Struct Funct 214:495-517. CrossRef Medline

Baddeley AD, Kopelman MD, Wilson BA (2003) The handbook of memory disorders. New York: Wiley.

Beckmann CFC, Jenkinson MM, Smith SMS (2003) General multilevel linear modeling for group analysis in FMRI. Neuroimage 20:12-21. CrossRef Medline

Bonnelle V, Ham TE, Leech R, Kinnunen KM, Mehta MA, Greenwood RJ, Sharp DJ (2012) Salience network integrity predicts default mode network function after traumatic brain injury. Proc Natl Acad Sci U S A 109:4690-4695. CrossRef Medline

Buckner RL, Andrews-Hanna JR, Schacter DL (2008) The brain's default network: anatomy, function, and relevance to disease. Ann N Y Acad Sci 1124:1-38. CrossRef Medline

Chen AC, Oathes DJ, Chang C, Bradley T, Zhou ZW, Williams LM, Glover GH, Deisseroth K, Etkin A (2013) Causal interactions between frontoparietal central executive and default-mode networks in humans. Proc Natl Acad Sci U S A 110:19944-19949. CrossRef Medline

Chiong W, Wilson SM, D'Esposito M, Kayser AS, Grossman SN, Poorzand P, Seeley WW, Miller BL, Rankin KP (2013) The salience network causally influences default mode network activity during moral reasoning. Brain 136:1929-1941. CrossRef Medline

Damoiseaux JS, Beckmann CF, Arigita EJ, Barkhof F, Scheltens P, Stam CJ, Smith SM, Rombouts SA (2008) Reduced resting-state brain activity in the "default network" in normal aging. Cereb Cortex 18:1856-1864. CrossRef Medline

Delis DC, Kaplan E, Kramer JH (2001) Delis-Kaplan executive function system. San Antonio, TX: The Psychological Corporation.

Dosenbach NU, Visscher KM, Palmer ED, Miezin FM, Wenger KK, Kang HC, Burgund ED, Grimes AL, Schlaggar BL, Petersen SE (2006) A core system for the implementation of task sets. Neuron 50:799-812. CrossRef Medline

Filippini N, MacIntosh BJ, Hough MG, Goodwin GM, Frisoni GB, Smith SM, Matthews PM, Beckmann CF, Mackay CE (2009) Distinct patterns of brain activity in young carriers of the APOE-epsilon4 allele. Proc Natl Acad Sci U S A 106:7209-7214. CrossRef Medline

Fransson P (2005) Spontaneous low-frequency BOLD signal fluctuations: an fMRI investigation of the resting-state default mode of brain function hypothesis. Hum Brain Mapp 26:15-29. CrossRef Medline

Friston KJ, Buechel C, Fink GR, Morris J, Rolls E, Dolan RJ (1997) Psychophysiological and modulatory interactions in neuroimaging. Neuroimage 6:218-229. CrossRef Medline

Gentry LR, Godersky JC, Thompson B (1988) MR imaging of head trauma: review of the distribution and radiopathologic features of traumatic lesions. AJR Am J Roentgenol 150:663-672. CrossRef Medline

Gusnard DA, Akbudak E, Shulman GL, Raichle ME (2001) Medial prefrontal cortex and self-referential mental activity: relation to a default mode of brain function. Proc Natl Acad Sci U S A 98:4259-4264. CrossRef Medline

Hua K, Zhang J, Wakana S, Jiang H, Li X, Reich DS, Calabresi PA, Pekar JJ, van Zijl PC, Mori S (2008) Tract probability maps in stereotaxic spaces: analyses of white matter anatomy and tract-specific quantification. Neuroimage 39:336-347. CrossRef Medline

Jenkinson M, Bannister P, Brady M, Smith S (2002) Improved optimization for the robust and accurate linear registration and motion correction of brain images. Neuroimage 17:825-841. CrossRef Medline

Kelly AM, Uddin LQ, Biswal BB, Castellanos FX, Milham MP (2008) Com- 
petition between functional brain networks mediates behavioral variability. Neuroimage 39:527-537. CrossRef Medline

Kim C, Johnson NF, Cilles SE, Gold BT (2011) Common and distinct mechanisms of cognitive flexibility in prefrontal cortex. J Neurosci 31:47714779. CrossRef Medline

Leech R, Sharp DJ (2014) The role of the posterior cingulate cortex in cognition and disease. Brain 137:12-32. CrossRef Medline

Leech R, Kamourieh S, Beckmann CF, Sharp DJ (2011) Fractionating the default mode network: distinct contributions of the ventral and dorsal posterior cingulate cortex to cognitive control. J Neurosci 31:3217-3224. CrossRef Medline

Leech R, Braga R, Sharp DJ (2012) Echoes of the brain within the posterior cingulate cortex. J Neurosci 32:215-222. CrossRef Medline

Leunissen I, Coxon JP, Geurts M, Caeyenberghs K, Michiels K, Sunaert S, Swinnen SP (2013) Disturbed cortico-subcortical interactions during motor task switching in traumatic brain injury. Hum Brain Mapp 34: 1254-1271. CrossRef Medline

Logan GD, Cowan WB, Davis KA (1984) On the ability to inhibit simple and choice reaction time responses: a model and a method. J Exp Psychol Hum Percept Perform 10:276-291. CrossRef Medline

Malec JF, Brown AW, Leibson CL, Flaada JT, Mandrekar JN, Diehl NN, Perkins PK (2007) The Mayo classification system for traumatic brain injury severity. J Neurotrauma 24:1417-1424. CrossRef Medline

Menon V, Uddin LQ (2010) Saliency, switching, attention and control: a network model of insula function. Brain Struct Funct 214:655-667. CrossRef Medline

Monsell S (2003) Task switching. Trends Cogn Sci 7:134-140. CrossRef Medline

O’Reilly JX, Woolrich MW, Behrens TE, Smith SM, Johansen-Berg H (2012) Tools of the trade: psychophysiological interactions and functional connectivity. Soc Cogn Affect Neurosci 7:604-609. CrossRef Medline

Raichle ME, MacLeod AM, Snyder AZ, Powers WJ, Gusnard DA, Shulman GL (2001) A default mode of brain function. Proc Natl Acad Sci U S A 98:676-682. CrossRef Medline

Reitan RM, Wolfson D (2004) The Trail Making Test as an initial screening procedure for neuropsychological impairment in older children. Arch Clin Neuropsychol 19:281-288. CrossRef Medline

Scheid R, Preul C, Gruber O, Wiggins C, von Cramon DY (2003) Diffuse axonal injury associated with chronic traumatic brain injury: evidence from $\mathrm{T}^{*}$-weighted gradient-echo imaging at $3 \mathrm{~T}$. AJNR Am J Neuroradiol 24:1049-1056. Medline

Seeley WW, Menon V, Schatzberg AF, Keller J, Glover GH, Kenna H, Reiss AL, Greicius MD (2007) Dissociable intrinsic connectivity networks for salience processing and executive control. J Neurosci 27:2349-2356. CrossRef Medline

Seeley WW, Crawford RK, Zhou J, Miller BL, Greicius MD (2009) Neurodegenerative diseases target large-scale human brain networks. Neuron 62:42-52. CrossRef Medline
Sharp DJ, Bonnelle V, De Boissezon X, Beckmann CF, James SG, Patel MC, Mehta MA (2010) Distinct frontal systems for response inhibition, attentional capture, and error processing. Proc Natl Acad Sci U S A 107: 6106-6111. CrossRef Medline

Sharp DJ, Beckmann CF, Greenwood R, Kinnunen KM, Bonnelle V, De Boissezon X, Powell JH, Counsell SJ, Patel MC, Leech R (2011) Default mode network functional and structural connectivity after traumatic brain injury. Brain 134:2233-2247. CrossRef Medline

Singh AK, Asoh H, Phillips S (2011) Optimal detection of functional connectivity from high-dimensional EEG synchrony data. Neuroimage 58 : 148-156. CrossRef Medline

Smith SM (2002) Fast robust automated brain extraction. Hum Brain Mapp 17:143-155. CrossRef Medline

Smith SM, Jenkinson M, Johansen-Berg H, Rueckert D, Nichols TE, Mackay CE, Watkins KE, Ciccarelli O, Cader MZ, Matthews PM, Behrens TE (2006) Tract-based spatial statistics: voxelwise analysis of multi-subject diffusion data. Neuroimage 31:1487-1505. CrossRef Medline

Smith SM, Fox PT, Miller KL, Glahn DC, Fox PM, Mackay CE, Filippini N, Watkins KE, Toro R, Laird AR, Beckmann CF (2009) Correspondence of the brain's functional architecture during activation and rest. Proc Natl Acad Sci U S A 106:13040-13045. CrossRef Medline

Smith SM, Jenkinson M, Woolrich MW, Beckmann CF, Behrens TEJ, Johansen-Berg H, Bannister PR, De Luca M, Drobnjak I, Flitney DE, Niazy RK, Saunders J, Vickers J, Zhang Y, De Stefano N, Brady JM, Matthews PM (2004) Advances in functional and structural MR image analysis and implementation as FSL. Neuroimage 23 [Suppl 1]:S208-S219.

Smith SM, Miller KL, Salimi-Khorshidi G, Webster M, Beckmann CF, Nichols TE, Ramsey JD, Woolrich MW (2011) Network modelling methods for FMRI. Neuroimage 54:875-891. CrossRef Medline

Squarcina L, Bertoldo A, Ham TE, Heckemann R, Sharp DJ (2012) A robust method for investigating thalamic white matter tracts after traumatic brain injury. Neuroimage 63:779-788. CrossRef Medline

Sridharan D, Levitin DJ, Menon V (2008) A critical role for the right frontoinsular cortex in switching between central-executive and default-mode networks. Proc Natl Acad Sci U S A 105:12569-12574. CrossRef Medline

Sudevan P, Taylor DA (1987) The cuing and priming of cognitive operations. J Exp Psychol Hum Percept Perform 13:89-103. CrossRef Medline

von Economo C, Koskinas G (1925) Die Cytoarchitectonik der Hirnrinde des erwachsenen Menschen. New York: Springer.

Wechsler D (1999) Wechsler Abbreviated Scale of Intelligence. San Diego: Academic.

Weissman DH, Roberts KC, Visscher KM, Woldorff MG (2006) The neural bases of momentary lapses in attention. Nat Neurosci 9:971-978. CrossRef Medline

Zuo XN, Kelly C, Adelstein JS, Klein DF, Castellanos FX, Milham MP (2010) Reliable intrinsic connectivity networks: test-retest evaluation using ICA and dual regression approach. Neuroimage 49:2163-2177. CrossRef Medline 